\title{
Marco normativo de la gestación por sustitución en México: desafíos internos y externos*
}

\section{María Mercedes Albornoz** Francisco López González***}

\section{RESUMEN}

La gestación por sustitución o maternidad subrogada es una práctica controvertida a la cual, tanto en México como en otros países, se recurre cada vez con más frecuencia. El marco normativo mexicano en esta materia es muy diverso; ha habido modificaciones locales recientes y es probable que pronto haya nuevos cambios a nivel nacional. Las normas juridicas mexicanas vigentes sobre el tema, y aquellas cuya adopción se discute actualmente en el Poder Legislativo, impactan e impactarian en casos internos, asi como en casos vinculados con ordenamientos juridicos extranjeros.

El objetivo de este artículo es presentar algunos de los rasgos primordiales del heterogéneo marco normativo de la gestación por sustitución en México, para identificar los desafios que el pais enfrenta en el orden interno y en el orden externo.

PALABRAS CLAVE: Gestación por sustitución, acuerdos de gestación por sustitución, maternidad subrogada, marco jurídico, México.

\section{ABSTRACT}

Substitute gestation or surrogate motherhood is a controversial practice increasingly often used in Mexico and in other countries as well. The Mexican regulatory framework in this area is very diverse. There have been recent legislative modifications at the local level and it is highly probable that new changes at the federal level will come soon. Mexican legal rules in force on the subject, and those that are currently being discussed in the legislative branch, do have and will have an impact in domestic cases, and also in cases that are linked to foreign legal systems.

The aim of this article is to present some of the paramount aspects of substitute gestation in Mexico, in order to identify the challenges that the country must face at the domestic and international levels.

KEY WORDS: Substitute gestation, surrogacy arrangements, surrogate motherhood, legal framework, Mexico.

\footnotetext{
* Artículo recibido el 27 de septiembre de 2016 y aceptado el 30 de octubre de 2016

"Profesora investigadora de la División de Estudios Jurídicos del Centro de Investigación y Docencia Económicas (CIDE), México. mercedes.albornoz@cide.edu

*** Profesor del Posgrado en Derecho de la Universidad Nacional de Autónoma de México (UNAM), México. flopezg@ derecho.unam.mx
} 


\section{SUMARIO}

1. Introducción

2. Necesidad de regulación clara

3. Un marco normativo heterogéneo

4. Desafíos internos y externos

5. Conclusiones

6. Bibliografía

\section{Introducción}

La gestación por sustitución, también conocida como maternidad subrogada, gestación subrogada, gestación por otro, alquiler de útero o de vientre, ${ }^{1}$ es una práctica controvertida, cuya utilización se ha incrementado en los últimos años. Actualmente se estima ${ }^{2}$ que al menos unos 20000 bebés nacen cada año por medio de gestación por sustitución ${ }^{3}$ y la cifra ha ido creciendo, tanto en el mundo como en México, con el aumento de la infertilidad.

Desde una perspectiva amplia, la gestación por sustitución es aquella que surge de un contrato, a título oneroso o gratuito, celebrado entre una persona física o una pareja de padres intencionales y una mujer, a fin de que ésta lleve a cabo la gestación de un embrión y que, cuando haya nacido el bebé, lo entregue al o a los padres intencionales. El niño nacido en estas circunstancias podría tener un vínculo biológico con uno o con ambos padres, o con ninguno, si interviniesen donantes de gametos. Asimismo, podría no tener vínculo biológico con la mujer gestante, o sí, en caso de que ella haya aportado su óvulo.

A partir de esa noción amplia es factible agregar diversas limitaciones que restringen el concepto de gestación por sustitución. Por ejemplo, que el contrato sólo pueda ser gratuito; los padres intencionales o comitentes únicamente puedan ser parejas, o bien solamente parejas heterosexuales, o parejas heterosexua-

\footnotetext{
1 Entre éstas y otras denominaciones, consideramos más apropiada la de "gestación por sustitución", puesto que pone el acento en la finalidad que persiguen las partes contratantes: que una persona sustituya a otra en la gestación. González Martín, Nuria y Albornoz, Maria Mercedes, "Aspectos transfronterizos de la gestación por sustitución", Anuario Mexicano de Derecho Internacional, volumen 16, México, enero-diciembre 2016. [Consulta: 8 de diciembre, 2016]. Disponible en: https://revistas.juridicas.unam.mx/index.php/derecho-internacional/article/view/524/784

${ }^{2}$ Se trata tan solo de una estimación, dada la dificultad para obtener datos precisos. Este problema se presenta también con respecto a la gestación por sustitución transfronteriza. Trimmings, Katarina y Beaumont, Paul (Eds.), International Surrogacy Arrangements. Legal Regulation at the International Level, Oxford, Hart Publishing, 2013, pp. 464-465.

${ }^{3}$ International Social Service, Call for Action 2016, Urgent need for regulation of the international surrogacy and artificial reproductive technologies. [Consulta: 8 de diciembre, 2016]. Disponible en http://www.iss-ssi.org/images/ Surrogacy/Call_for_Action2016.pdf
} 
les casadas, o que sólo se pueda recurrir a la gestación por sustitución cuando por razones médicas la mujer de la pareja no tenga posibilidad de gestar.

De estos ejemplos quedarían excluidos, respectivamente, los contratos de gestación por sustitución onerosos Den los cuales la mujer gestante reciba una suma de dinero que exceda los gastos a los que ella deba hacer frente como consecuencia de la gestación $\square$; aquéllos con un único padre intencional o una única madre intencional; los contratos en los cuales los padres intencionales sean parejas homosexuales, o parejas heterosexuales que no hayan contraído matrimonio; también los contratos con padres intencionales heterosexuales, cuando la motivación para recurrir a la gestación por sustitución no sea estrictamente médica.

En la actualidad (finales de 2016) no existe un tratado internacional, ni de alcance universal ni de alcance regional, que regule la gestación por sustitución. ${ }^{4}$ Por lo tanto, México no está vinculado por ningún instrumento internacional específico sobre esta modalidad de reproducción humana asistida. Pero, en el orden interno, existen en el país ciertas reglas en la materia que se caracterizan por su falta de homogeneidad. En efecto, la diversidad del marco normativo mexicano sobre gestación por sustitución y algunos aspectos del contenido de la regulación existente o que se encuentra en fase de tratamiento legislativo, plantean para México desafíos tanto internos como externos. El objetivo de este artículo es identificarlos.

\section{Necesidad de regulación clara}

El surgimiento y la propagación de la gestación por sustitución se deben por una parte a los avances científicos, dado que esta práctica generalmente implica el recurso a técnicas de reproducción humana asistida. Por otra, al incremento de la infertilidad vinculada con la cada vez más habitual postergación de la decisión de procrear y a la proliferación de estructuras familiares novedosas, ${ }^{5}$ como las monoparentales y las homoparentales.

Ahora bien, si por un lado existen el derecho a formar una familia y la voluntad de incorporar uno o más miembros a la familia mediante la gestación por sustitución, por el otro hay una mujer que presta su cuerpo para llevar a cabo la gestación y el parto, además de las circunstancias en que nacen el niño

\footnotetext{
"Véase González Martin, Nuria y Albornoz, Maria Mercedes, "Aspectos transfronterizos de la gestación por sustitución", Anuario Mexicano de Derecho Internacional, volumen 16, México, enero-diciembre 2016. [Consulta: 8 de diciembre, 2016]. Disponible en: https://revistas.juridicas.unam.mx/index.php/derecho-internacional/article/view/524/784 ${ }^{5}$ HeRreRA, Marisa, "Sobre familias en plural. Reformar para transformar", Revista Jurídica Universidad de Ciencias Empresariales y Sociales, No. 17, 2013, pp. 105-132. [Consulta: 8 de diciembre, 2016]. Disponible en http://dspace. uces.edu.ar:8180/xmlui/bitstream/handle/123456789/2142/Sobre_familias_Herrera.pdf?sequence=1
} 
o los niños. Los intereses de todos los sujetos involucrados son merecedores de protección jurídica. No obstante, la gestación por sustitución suele ser cuestionada porque podría propiciar la explotación de jóvenes mujeres en situación de vulnerabilidad como madres gestantes, la mercantilización de la filiación y el tráfico de niños así nacidos. ${ }^{6}$

También genera preocupación la manera en que corresponde determinar la filiación de estos menores, cuando se advierte que la máxima mater semper certa est ha sido rebasada por la realidad actual. ${ }^{7}$

$\mathrm{Al}$ menos dos cuestiones relevantes subyacen en este tema, desde la óptica del legislador mexicano. La primera consiste en decidir si es necesario regular la gestación por sustitución, sea de manera positiva o negativa, permitiéndole o negándole la producción de efectos jurídicos o, si es preferible, guardar silencio al respecto. La segunda alude a si es posible regular la figura de manera que se logre garantizar el derecho a la procreación, sin conculcar la libertad de las mujeres gestantes ni el interés superior de la niñez.

Con respecto a la primera cuestión, es preciso recordar que esta práctica existe en el mundo, no solamente en el extranjero, sino también en México. ${ }^{8}$ El silencio o la prohibición legislativa pueden conducir a que de todos modos se lleve a cabo al margen de la ley, sin garantías para ninguna de las partes, ni para los niños nacidos en tales circunstancias. ${ }^{9}$ Dados los potenciales conflictos que entraña su desarrollo en la clandestinidad, es importante que México cuente con una regulación clara de la institución.

La Corte Interamericana de Derechos Humanos profundiza en la necesidad de legislar la materia sin prohibirla. Considera el "útero subrogado" como una de las técnicas de reproducción asistida, es decir, como uno de los "tratamientos médicos que se utilizan para ayudar a las personas y parejas infértiles a lograr un embarazo" ${ }^{10}$ y reconoce que el derecho de acceder a

${ }^{6}$ Algunos textos advierten acerca de los riesgos éticos de la gestación por sustitución, por ejemplo, el de López Guzmán, José y Aparisi Miralles, Ángela, "Aproximación a la problemática ética y jurídica de la maternidad subrogada", Cuadernos de Bioética, vol. 23, No. 78, mayo-agosto, 2012, pp. 253-267. [Consulta: 8 de diciembre, 2016]. Disponible en http://aebioetica.org/revistas/2012/23/78/253.pdf

${ }^{7}$ Como lo señala una autora argentina, el principio de que "la madre siempre es cierta" se encuentra en crisis. Lamm, Eleonora, "Gestación por sustitución", InDret. Revista para el Análisis del Derecho, marzo, 2012, p. 5. [Consulta: 8 de

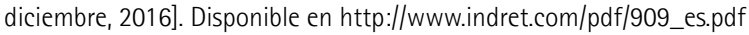

${ }^{8}$ Salgado, SARA, "La gestación subrogada en México: leyes en cada estado y precios", Babygest, septiembre, 2016. [Consulta: 8 de diciembre, 2016]. Disponible en http://www.babygest.es/mexico/

${ }^{9}$ La situación sería similar a la que se da en los sistemas jurídicos que prohíben el aborto.

${ }^{10}$ Corte Interamericana de Derechos Humanos, "Caso Artavia Murillo y otros (fecundación in vitro) vs. Costa Rica", 28 de noviembre, 2012, p. 21, párrafo 63. [Consulta: 8 de diciembre, 2016]. Disponible en http://www.corteidh.or.cr/docs/ casos/articulos/seriec_257_esp.pdf 
tales técnicas guarda relación con el derecho a la vida privada y la libertad reproductiva. ${ }^{11}$

En cuanto a la segunda cuestión subyacente, se entiende que, incluso sin intención de incentivar a la población a gestar por esta vía, es posible regularla y hallar un equilibrio entre el derecho a la procreación, la libertad de la gestante y el interés superior de los niños. La gran pregunta es cómo. A fin de aportar elementos a la discusión, se requiere identificar ciertos desafíos concretos a los cuales, más temprano que tarde, México habrá de hacer frente.

\section{Un marco normativo heterogéneo}

El artículo 4 constitucional establece que en los Estados Unidos Mexicanos toda persona tiene derecho a decidir sobre el número y espaciamiento de sus hijos de manera libre, responsable e informada. Esto puede entenderse como el fundamento, al más alto nivel dentro de la jerarquía mexicana de leyes, del derecho a la libre reproducción. Los alcances de dicha disposición se potencian con lo prescrito en el artículo 17 de la Convención Americana sobre Derechos Humanos, de la cual México es parte. Ésta reconoce el derecho de las personas a fundar una familia si tienen la edad y las condiciones que requieren las leyes internas para ello.

De esta manera, si en México las personas mayores de edad tienen derecho a fundar una familia y decidir sobre el número de hijos que conformarán esa familia, así como el momento de concebirlos y tenerlos, la gestación por sustitución, como método de reproducción humana técnicamente asistida, puede entenderse como uno de varios componentes del derecho a la libre reproducción.

Adicionalmente, en algunas entidades federativas del país se ha legislado la materia y en varias de ellas ha habido reformas en el último año. Tales reglas en ocasiones presentan disimilitudes entre sí, conformando un marco normativo heterogéneo. Por un lado, permiten la gestación por sustitución los estados de Tabasco y Sinaloa; por otro lado, la prohíben en San Luis Potosí y Querétaro. Asimismo, cabe señalar que hace unos años se aprobó la "Ley de Maternidad Subrogada" para el Distrito Federal, ${ }^{12}$ sin embargo, no ha sido publicada ofi-

\footnotetext{
${ }^{11}$ Corte Interamericana de Derechos Humanos, "Caso Artavia Murillo y otros (fecundación in vitro) vs. Costa Rica", 28 de noviembre, 2012, p. 49, párrafo 150. [Consulta: 8 de diciembre, 2016]. Disponible en http://www.corteidh.or.cr/docs/ casos/articulos/seriec_257_esp.pdf

${ }^{12}$ La iniciativa fue aprobada por la Asamblea Legislativa el 30 de noviembre de 2010. Contreras Julían, Maricela, Iniciativa con proyecto de decreto por el que se expide la Ley de Maternidad Subrogada del Distrito Federal, 26 de noviembre de 2009. [Consulta: 8 de diciembre, 2016]. Disponible en http://www.aldf.gob.mx/archivo-3f7e1195feb6a2a6fc616d1cef522305.pdf
} 
cialmente ni se ha convertido en derecho positivo. Además, Coahuila contaba con una disposición prohibitiva muy similar a la de San Luis Potosí, pero fue derogada el 15 de diciembre de 2015.

En el Congreso de la Unión se ha dado impulso a diversas iniciativas de ley en esta materia. En caso de prosperar, podrían contribuir a la homogeneización de la normatividad dentro del país. Aquellas dos cuyo trámite parlamentario más ha avanzado (en adelante, "las iniciativas") proponen abordar el tema en la Ley General de Salud y las presentaron dos legisladoras del Partido Revolucionario Institucional: una en el Senado y la otra en la Cámara de Diputados.

La primera iniciativa fue presentada por la senadora Mely Romero Celis y otros miembros del Grupo Parlamentario del PRI el 13 de octubre de 2015, para agregarle a la Ley General de Salud los artículos 61 Ter y 462 Ter. Esta iniciativa sufrió cambios importantes, pero luego fue aprobada por el Senado el 26 de abril de 2016 y remitida a la Cámara de Diputados para su discusión. ${ }^{13} \mathrm{La}$ segunda iniciativa estuvo a cargo de la diputada Sylvana Beltrones Sánchez, quien reforma y adiciona diversas disposiciones a la Ley General de Salud. Fue presentada en la Cámara de Diputados el 1 de marzo de 2016. ${ }^{14}$

\section{Desafíos internos y externos}

México enfrenta desafíos internos y externos en materia de gestación por sustitución. Aquí se intenta contribuir al debate acerca de cómo sería conveniente regularla, identificando algunos de sus desafíos.

Un primer desafío interno consiste en dilucidar dónde sería mejor ubicar la gestación por sustitución, dentro del sistema mexicano de competencias legislativas. ${ }^{15}$ En este sentido, llama la atención que las iniciativas surjan dentro de la regulación de la salud. Si bien es evidente que la institución tiene implicaciones en el ámbito de la salud, también produce efectos de derecho civil en aspectos como la filiación, ${ }^{16}$ la patria potestad, los alimentos y las sucesiones. Y la regulación de la materia civil recae dentro del ámbito estatal, lo cual, de

\footnotetext{
${ }^{13}$ Senado de la República, Gaceta del Senado, XLIII, 1SPO-132/62316, 26 de abril, 2016. [Consulta: 8 de diciembre, 2016]. Disponible en http://www.senado.gob.mx/index.php?ver $=s p \& t m n=2 \& t s m=2 \& t i d=62316$

${ }^{14}$ Beltrones Sánchez, Sylvana, Gaceta Parlamentaria, 4479-IV, 1 de marzo, 2016. [Consulta: 8 de diciembre, 2016]. Disponible en http://gaceta.diputados.gob.mx/Black/Gaceta/Anteriores/63/2016/mar/20160301-IV/Iniciativa-12.html

${ }^{15}$ Conocido como "sistema de competencias residuales" por la fórmula utilizada en el artículo 124 de la Constitución Política de los Estados Unidos Mexicanos, el cual señala que todas aquellas facultades no expresamente otorgadas a la Federación se entienden reservadas a los estados.

${ }^{16}$ Adicionalmente, y como consecuencia de la filiación, la gestación por sustitución tiene efectos en materia de atribución de nacionalidad.
} 
entrada, implica un problema con respecto a la uniformidad del tratamiento de la gestación por sustitución en la legislación mexicana.

Tal vez se haya optado por regular la gestación por sustitución a través de la legislación en materia de salud porque ésta es de carácter general y eso significa que impacta por igual en las esferas federal, estatal y municipal. Así, en gran medida, la uniformidad podría conseguirse por esta vía; pero sólo en cuanto a los aspectos meramente contractuales de la gestación por sustitución y no en lo atinente al estado civil y a sus consecuencias en materia familiar.

Otro desafío interno es definir si, más allá de la manera en que se la denomine, la institución comprenderá tanto el caso en que la mujer gestante aporte su útero sin tener vínculo biológico con el niño, como en el que aporte su propio material genético. Este último supuesto, regulado tanto en Tabasco como en Sinaloa, está excluido en las iniciativas.

Un importante desafío externo es evitar que México se siga percibiendo internacionalmente como un destino de turismo reproductivo. Con la intención de atacar este problema Qque ya se había presentado en la práctica, especialmente en TabascoL, tanto las iniciativas como la legislación tabasqueña y la sinaloense exigen que los padres intencionales sean ciudadanos mexicanos. Se prohíbe el acceso a la gestación por sustitución a todo extranjero, incluso al extranjero residente en México; pero se le permite a cualquier mexicano, incluso si reside en el exterior.

Sin embargo, se estima que, para lograr el resultado deseado por el legislador, sería más apropiado tomar como factor relevante el país de residencia, en lugar de la nacionalidad de los padres intencionales. Esta solución contribuiría a reducir problemas de derecho internacional privado ${ }^{17}$ y evitaría una discriminación por nacionalidad que eventualmente podría dar lugar a litigios sobre la constitucionalidad de la restricción.

El desafío interno de determinar quiénes pueden acceder a la gestación por sustitución como padres intencionales está directamente relacionado con el anterior y es más amplio. Tanto en la iniciativa de Romero como en la legislación de Tabasco y en la de Sinaloa, la gestación por sustitución está abierta para las parejas heterosexuales, en las cuales la mujer por imposibilidad física o por motivos médicos no puede gestar.

\footnotetext{
${ }^{17}$ Tales como el reconocimiento de actas de nacimiento expedidas en el extranjero; la determinación de la jurisdicción internacionalmente competente y del derecho aplicable para dilucidar controversias acerca de la gestación por sustitución con elementos de extranjería. Asimismo, el reconocimiento de las relaciones de filiación que surjan con la gestación por sustitución llevada a cabo en un pais diferente de donde residen los padres intencionales y el destino al que llevan, o quieren llevar, a vivir al niño.
} 
Sin embargo, se restringe el acceso cuando las razones para recurrir a esta práctica son otras. Nótese que en la iniciativa de Beltrones el único motivo admitido es la infertilidad de los solicitantes de técnicas de reproducción humana médicamente asistida. Mientras, en las normas de Tabasco y Sinaloa, así como en la iniciativa de Romero, las parejas heterosexuales que contraten la gestación por sustitución pueden ser cónyuges o concubinos. Pero no se permite que sean padres intencionales las personas solteras que no estén en una relación de concubinato, ni los concubinos o cónyuges de un mismo sexo, sin importar el motivo por el que tengan voluntad de procrear.

En la iniciativa de Beltrones, las parejas que soliciten técnicas de reproducción asistida deben estar unidas en matrimonio y no haberse separado ni haber presentado solicitud de separación, anulación o disolución del matrimonio, ni de cesación de los efectos civiles matrimoniales. Adicionalmente, esta última iniciativa exige que exista un vínculo de parentesco entre alguna de las dos personas de la pareja y la mujer gestante, restricción que no está presente en los otros cuerpos de normas considerados.

Otro desafío de orden interno es el de establecer controles para evitar la comercialización con la vida humana. Es factible ver como comercialización la admisión del carácter oneroso del contrato de gestación por sustitución. Éste está permitido como una de las modalidades contractuales en Sinaloa (subrogación onerosa). En Tabasco, en cambio, la ausencia de prohibición expresa al respecto autoriza a inferir que sería posible pactar una retribución para la mujer gestante.

Al contrario, las iniciativas exigen que el contrato sea a título gratuito. El pago de una contraprestación que exceda la "compensación de gastos médicos y otros derivados del embarazo, parto, post-parto y puerperio” está prohibido en la iniciativa de Romero. ${ }^{18}$ La iniciativa de Beltrones establece que a la mujer gestante la contraparte deberá cubrirle todos los gastos que se originen como consecuencia del procedimiento, tales como gastos anteriores $\mathrm{y}$ posteriores al parto y los que sean necesarios para garantizar su salud y 180 correcta recuperación. ${ }^{19}$

No obstante, también sería posible sostener que la admisión de una contraprestación a la gestante no implica más que la retribución por un servicio prestado, y no significa que necesariamente haya comercialización ni con la

\footnotetext{
${ }^{18}$ Artículo 61 Ter de la iniciativa, cuyo artículo 462 Ter además admite la subrogación "sin fines de lucro" y para el caso contrario establece sanciones.

${ }^{19}$ Artículo 71 Bis 10 de la iniciativa.
} 
vida del niño ni con el cuerpo de la madre. ${ }^{20}$ Desde esta perspectiva, se supone que la mercantilización de esta técnica de reproducción humana asistida suele venir dada, más bien, por la participación inescrupulosa de terceros. Así, se considera más acuciante establecer controles o restricciones en cuanto a la conducta de agencias de intermediación, de médicos y personal auxiliar. Tanto la legislación de Tabasco como la de Sinaloa y las iniciativas instituyen sendos sistemas de control, los cuales tienden a evitar que se comercialice con la vida.

Otro desafío íntimamente vinculado con el anterior es la determinación del perfil de la mujer gestante y la protección de sus derechos. En este sentido, es indispensable que la mujer que llevará a cabo la gestación goce de plena salud física y psíquica. También se estima relevante que, desde antes de firmar el contrato de gestación por sustitución, cuente con toda la información sobre esta práctica y sus consecuencias, y que sea capaz de comprenderla cabalmente.

Hay además un desafío externo que México ya enfrenta: cómo coadyuvar a la solución de los casos internacionales de gestación por sustitución que se han presentado y que se presentarán en el futuro. Porque, aunque se limitara el acceso a la gestación por sustitución únicamente a nacionales mexicanos y esa medida redujera la cantidad de casos transfronterizos, no los excluiría totalmente.

Este desafío comprende la participación en discusiones acerca de la viabilidad y el contenido de un posible nuevo instrumento internacional, ${ }^{21}$ así como su futura adopción. Abarca también la legislación de fuente interna y se extiende inclusive hasta la puesta en marcha de todo el andamiaje de cooperación internacional entre autoridades.

Finalmente, una vez que se haya promulgado un determinado régimen normativo para la gestación por sustitución, el reto es su implementación. En otras palabras, la instrumentación y la viabilidad. Si la regulación es restrictiva, la puesta en marcha de controles por parte de la autoridad es un punto crucial para que el sistema no sea desvirtuado en la práctica.

\footnotetext{
${ }^{20}$ Nótese que el Código Familiar del Estado de Sinaloa determina la nulidad del instrumento cuyas cláusulas atenten contra el interés superior del niño y la dignidad humana, o contravengan el orden social y el interés público (artículo 288, III y IV) y, sin embargo, admite que el contrato tenga carácter oneroso (artículo 284, III).

${ }^{21}$ México participa en los trabajos del grupo de expertos en filiación y gestación por sustitución que, bajo los auspicios de la Conferencia de La Haya de Derecho Internacional Privado, se reunió por primera vez en febrero de 2016. La próxima reunión tendrá lugar a finales de enero y principios de febrero de 2017.
} 


\section{Conclusiones}

La gestación por sustitución es una realidad ante la cual el legislador mexicano debe reaccionar. Se requiere contar con un nuevo marco normativo Dhomogéneo en lo esencial $\square$ que regule esta práctica, de manera tal que se alcance un equilibrio entre el derecho de los padres intencionales, la libertad de la mujer gestante y la defensa del interés superior de los niños que nacen como resultado de la gestación por sustitución.

En cuanto al diseño del contenido de las normas, es preciso tomar en cuenta que, en esta materia, México se enfrenta, al menos, a los siguientes desafíos: decidir dónde ubicar la gestación por sustitución, dentro del sistema mexicano de competencias; definir si ésta comprenderá también los casos en los cuales la mujer gestante aporta su propio material genético; revertir la percepción internacional de México como destino de turismo reproductivo; determinar quiénes pueden ser padres intencionales; establecer controles para que no se comercialice con la vida humana; determinar el perfil de la mujer gestante y cómo proteger sus derechos; decidir cómo coadyuvar a la solución de los casos transfronterizos de gestación por sustitución; llevar a cabo la instrumentación y la viabilidad del régimen normativo sin desnaturalizarlo.

La tarea de regular la gestación por sustitución debe ser emprendida con suma diligencia, cuidando no introducir disposiciones discriminatorias que sean tierra fértil para el litigio sobre su constitucionalidad. Se trata de una labor compleja que requiere creatividad legislativa y voluntad política. Es urgente que México tome cartas en el asunto.

\section{Bibliografía}

Beltrones Sánchez, Silvana, Gaceta Parlamentaria, 4479-IV, 1 de marzo, 2016. [Consulta: 8 de diciembre, 2016]. Disponible en http://gaceta.diputados.gob.mx/ Black/Gaceta/Anteriores/63/2016/mar/20160301-IV/Iniciativa-12.html

Contreras Julián, Maricela, Iniciativa con proyecto de decreto por el que se expide la Ley de Maternidad Subrogada del Distrito Federal, 26 de noviembre de 2009. [Consulta: 8 de diciembre, 2016]. Disponible en http://www.aldf.gob. $\mathrm{mx} /$ archivo-3f7e1195feb6a2a6fc616d1cef522305.pdf

Corte Interamericana de Derechos Humanos, "Caso Artavia Murillo y otros (fecundación in vitro) vs. Costa Rica”, 28 de noviembre, 2012, p. 21, párrafo 63. [Consulta: 8 de diciembre, 2016]. Disponible en http://www.corteidh.or.cr/docs/ casos/articulos/seriec_257_esp.pdf

González Martín, Nuria y Albornoz, María Mercedes, "Aspectos transfronterizos de la gestación por sustitución”, Anuario Mexicano de Derecho Internacio- 
nal, volumen 16, México, enero-diciembre 2016. [Consulta: 8 de diciembre, 2016]. Disponible en: https://revistas.juridicas.unam.mx/index.php/derecho-internacional/article/view/524/784

HERRERA, MARISA, “Sobre familias en plural. Reformar para transformar”, Revista Jurídica Universidad de Ciencias Empresariales y Sociales, No. 17, 2013, pp. 105-132. [Consulta: 8 de diciembre, 2016]. Disponible en http://dspace.uces. edu.ar:8180/xmlui/bitstream/handle/123456789/2142/Sobre_familias_Herrera.pdf?sequence $=1$

International Social Service, Call for Action 2016, Urgent need for regulation of the international surrogacy and artificial reproductive technologies. [Consulta: 8 de diciembre, 2016]. Disponible en http://www.iss-ssi.org/images/Surrogacy/Call_for_Action2016.pdf

Lamm, Eleonora, “Gestación por sustitución”, InDret. Revista para el Análisis del Derecho, marzo, 2012, p. 5. [Consulta: 8 de diciembre, 2016]. Disponible en http://www.indret.com/pdf/909_es.pdf

López Guzmán, José y Aparisi Miralles, Ángela, “Aproximación a la problemática ética y jurídica de la maternidad subrogada”, Cuadernos de Bioética, vol. 23, No. 78, mayo-agosto, 2012, pp. 253-267. [Consulta: 8 de diciembre, 2016]. Disponible en http://aebioetica.org/revistas/2012/23/78/253.pdf

SAlgado, SARA, "La gestación subrogada en México: leyes en cada estado y precios", Babygest, septiembre, 2016. [Consulta: 8 de diciembre, 2016]. Disponible en http://www.babygest.es/mexico/

Senado de la República, Gaceta del Senado, XLIII, 1SPO-132/62316, 26 de abril, 2016. [Consulta: 8 de diciembre, 2016]. Disponible en http://www.senado.gob.mx/ index.php?ver=sp\&tmn=2\&tsm=2\&tid=62316

Trimmings, Katarina y Beaumont, Paul, International Surrogacy Arrangements. Legal Regulation at the International Level, Oxford, Hart Publishing, 2013. 
